\title{
Does laparoscopic liver resection for colorectal liver metastases provide a true survival benefit compared with open liver resection?
}

\author{
Shinichi Akahoshi ${ }^{1}$, Toru Beppu ${ }^{1}$, Yasuhisa Mori ${ }^{2}$, Koichi Kinoshita ${ }^{1}$, Nobutaka Sato ${ }^{1}$, Ken Kikuchi ${ }^{3}$ \\ ${ }^{1}$ Department of Surgery, Yamaga City Medical Center, Kumamoto, Japan; ${ }^{2}$ Department of Surgery and Oncology, Graduate School of Medical \\ Sciences, Kyushu University, Fukuoka, Japan; ${ }^{3}$ Adviser, Sakurajuji Hospital, Kumamoto, Japan \\ Correspondence to: Toru Beppu, MD, PhD, FACS. Department of Surgery, Yamaga City Medical Center, 511 Yamaga, Kumamoto 861-0593, Japan. \\ Email: tbeppu@yamaga-mc.jp. \\ Comment on: Syn NL, Kabir T, Koh YX, et al. Survival Advantage of Laparoscopic Versus Open Resection For Colorectal Liver Metastases A Meta- \\ analysis of Individual Patient Data From Randomized Trials and Propensity-score Matched Studies. Ann Surg 2019. [Epub ahead of print].
}

Submitted Dec 18, 2019. Accepted for publication Dec 30, 2019.

doi: 10.21037/hbsn.2020.01.01

View this article at: http://dx.doi.org/10.21037/hbsn.2020.01.01

The recent Southampton consensus guideline and two previous international consensus meetings held in Louisville and Morioka in 2008 and 2014, respectively have recommended laparoscopic liver resection (LLR) as a standard procedure for selected patients with colorectal liver metastases (CRLM) (1-3). The Oslo-CoMet and LapOpHuva studies are the only two randomized controlled trials (RCTs) that have provided solid evidence showing that LLR is a safer, less invasive, and more costeffective approach with higher health-related quality of life and oncological outcomes equivalent to those of open liver resection (OLR) for patients with CRLM (4-6). Many cohort studies that used propensity score matching (PSM) and their meta-analyses have demonstrated decreased intraoperative blood loss, blood transfusion rate, and morbidity; shorter hospital stays; and improved postoperative quality of life in LLR patient groups than in OLR groups (7-9). Additionally, long-term outcomes have been found to be comparable between these two treatment groups.

We deeply congratulate Dr. Syn and his colleagues for publishing "Survival advantage of laparoscopic versus open resection for colorectal liver metastases: A metaanalysis of individual patient data from randomized trials and propensity score-matched studies" in Annals of Surgery, 2019 (10). To the best of our knowledge, this is the first study to use an individual participant data (IPD) metaanalysis of that focused on the oncological efficacy of LLR and OLR for patients with CRLM. Of course, RCTs and their meta-analyses provide the highest level of evidence; however, PSM has been widely adopted as an established statistical method for comparison of different treatments with minimized selection bias (11). Therefore, the authors collected 3,148 participants from RCTs and PSM studies in this paper. In fact, the risk bias in the two RCTs was low in more than half of the domains investigated. Ten out of 13 PSM studies were judged as moderate to high quality by the Newcastle-Ottawa checklist. An IPD meta-analysis is a unique type of meta-analysis. Conventional meta-analyses use the entire mass data set of patients, i.e., the mean survival time and standard deviation. In contrast, this novel IPD meta-analysis used survival data of individual patients recreated from the published Kaplan-Meier curves in each paper. Frequentist and Bayesian survival models considering random effects and non-proportional hazards were used to compare the overall survival of patients who underwent LLR and OLR. We think that these data were supported by reasonable and strict statistical methods; however, to validate this methodology, a detailed comparative study of estimated data and realistic individual data would be required.

Surprisingly, this novel meta-analysis of high-quality studies indicated an unpredicted positive survival benefit in favor of LLR over OLR for CRLM [stratified hazard ratio $(\mathrm{HR})=0.853 ; 95 \%$ confidence interval (CI), 0.754-0.965; $\mathrm{P}=0.0114]$. A stratified Cox model was used to calculate this HR from the primary analysis. Similarly, both the marginal and gamma-frailty models showed statistically significant 
HRs (range, 0.85-0.88) in favor of the LLR. In previous reports, one meta-analysis of PSM studies demonstrated significantly better 3-year overall survival for LLR than for OLR (odds ratio, 1.37; 95\% CI, 1.11-1.69) (9). Another PSM study of CRLM patients who underwent simultaneous liver and colorectal resection showed significantly better overall survival for LLR than for OLR (HR, 0.56; $95 \%$ CI, 0.36-0.87) (8). Additionally, there was clear evidence of time-varying effects in which the magnitude of HRs increased over time.

Some papers have proposed a 5- or 10-year milestone for defining "cured" in CRLM patients after liver resection. Therefore, to account for the existence of a subgroup of patients who no longer contribute to calculate the hazard, cure models were created by using the Weibull mixture or flexible parametric cure models. Consequently, the fractions of long-term survivors were estimated to be $47.4 \%$ in the LLR group and $18.0 \%$ in the OLR group (Supplementary Figure S4). At the 10-year follow-up, the restricted mean survival time was 8.6 months longer in the LLR group $(\mathrm{P}<0.0001)$. Additionally, in a subgroup analysis, the survival benefit was also detectable in elderly patients (defined as patients $\geq 65$ years or $\geq 70$ years) from two studies ( $n=608$ ); the median survival time was 53.1 months for LLR and 44.9 months for OLR, with a longer 3-year mean life expectancy in the elderly patients.

The most important discussion point of this IPD metaanalysis concerns the question of why LLR provided better overall survival than that of OLR. The Oslo-CoMet RCT (12) emphasized three possible explanations for this phenomena: first, a reduced inflammatory response to accelerate proliferation and migration of cancer cells caused by lower levels of interleukin-6, C-reactive protein, and macrophage inflammatory protein- $1 \mathrm{~b}$; second, reduced release of high-mobility group box 1 as a potentially oncogenic protein and; third, a lower level of cell-free DNA as a marker of tissue damage and intravascular disseminated cancer cells.

In some previous RCTs and PSM studies that compared LLR and OLR for CRLM, lower postoperative complication rates, less blood loss, and lower blood transfusion rates in LLR have been reported (4,5,7-9). Greater morbidity, blood loss amount, and blood transfusion rate are well-known poor prognostic factors in patients with CRLM who undergo liver resection (13). The expected lesser invasiveness of LLR can result in better prognosis. Further, LLR is often used as a nonanatomical and parenchymal-sparing resection, which has been shown to be associated with improved survival (14) possibly by lowering the risk of post-surgical hepatic insufficiency.

Repeat liver resection is essential to achieve long-term survival after liver resection for CRLM. At the second or additional liver resection, lower dense adhesions after the initial LLR may also be associated with fewer postoperative complications and lower blood loss amounts after repeat hepatectomies and hence improved survival outcomes. Compared with repeat OLR, repeat LLR can provide shorter operation times, higher R0 resection rates, and shorter hospital stays (15). After initial LLR, repeat LLR tends to be selected more often than OLR.

Postoperative chemotherapy has been shown to be effective for improving disease-free and overall survival of patients with CRLM who underwent liver resection (16). Chemotherapy has been recommended to start within 2 months after resection of stage III colon cancer. The interval to adjuvant chemotherapy after CRLM has been reported to be shorter after LLR than after OLR (17).

To further improve long-term survival in LLR, the findings discussed below should be considered. A systematic review showed that conversion rates from LLR to OLR ranged from $4.8 \%$ to $9.8 \%$ (18). It has been clearly reported that patients who had an emergency conversion secondary to an unfavorable intraoperative event experienced worse outcome, such as intraoperative massive bleeding or injury to an adjacent organ, than the outcomes of patients who received an elective conversion (19). To obtain better longterm survival in patients planning to undergo LLR, strict preoperative evaluation and precise operative technique is required. Recently, remnant liver ischemia was found to be associated with poor recurrence-free and cancer-specific survival (20). High-quality anatomical resection might be preferable, even if the resection area is smaller than one segment. Fluorescence-guided surgery is one solution for completing anatomical resection without an extensive ischemic area of the remnant liver.

The last question concerns why Kaplan-Meier curves of overall survival in the pooled cohort (Figure 3A) were similar until the 5-year point in LLR and OLR patients, after which the curves gradually separated. There is a possibility of biases because of the small number of papers that had sufficiently long observation periods $>5$ years or a sufficient number of patients after 5 years. A definitive dilemma remains because the details of the background characteristics or recurrence data are unclear in this IPD meta-analysis. Beppu T, the corresponding author for this editorial, reported a PSM study with the largest number 
of CRLM patients in 2015 (7). The original overall survival curve of the Beppu study is shown in figure 2, and the survival curve is very similar to that of the pooled cohort. The hypothesis for the difference in survival rates in the second half of the observation period between the two groups includes the low rates of early recurrence or fewer life-shortened recurrence patterns in LLR. Earlier induction of adjuvant chemotherapy, higher rates of repeated liver resection, and preservation of liver function may also improve the survival rate in the second half. However, Beppu et al. reported no significant differences in recurrence-free survival, intervals to recurrence, and recurrence patterns between LLR and OLR (7).

The authors concluded that LLR is at least not inferior to the standard OLR from a conservative viewpoint. This conclusion may be because they observed no differences in the survival data of the integrated analysis of the two RCTs. However, we believe that this IPD meta-analysis clearly demonstrates a possible significant long-term survival benefit of LLR over OLR for CRLM. Unfortunately, the survival data after 5 years is insufficient from each of the cited papers in this IPD meta-analysis; therefore, reevaluation of the survival data with a longer observation time is strongly recommended.

\section{Acknowledgments}

Funding: None.

\section{Footnote}

Provenance and Peer Review: This article was commissioned by the editorial office, Hepatobiliary Surgery and Nutrition. The article did not undergo external peer review.

Conflicts of Interest: All authors have completed the ICMJE uniform disclosure form (available at http://dx.doi. org/10.21037/hbsn.2020.01.01). The authors have no conflicts of interest to declare.

Ethical Statement: The authors are accountable for all aspects of the work in ensuring that questions related to the accuracy or integrity of any part of the work are appropriately investigated and resolved.

Open Access Statement: This is an Open Access article distributed in accordance with the Creative Commons Attribution-NonCommercial-NoDerivs 4.0 International
License (CC BY-NC-ND 4.0), which permits the noncommercial replication and distribution of the article with the strict proviso that no changes or edits are made and the original work is properly cited (including links to both the formal publication through the relevant DOI and the license). See: https://creativecommons.org/licenses/by-nc-nd/4.0/.

\section{References}

1. Abu Hilal M, Aldrighetti L, Dagher I, et al. The Southampton Consensus Guidelines for Laparoscopic Liver Surgery: From Indication to Implementation. Ann Surg 2018;268:11-8.

2. Buell JF, Cherqui D, Geller DA, et al. World Consensus Conference on Laparoscopic Surgery. The international position on laparoscopic liver surgery: The Louisville Statement, 2008. Ann Surg 2009;250:825-30.

3. Wakabayashi G, Cherqui D, Geller DA, et al. Recommendations for laparoscopic liver resection: A report from the 2nd International Consensus Conference Held in Morioka. Ann Surg 2015;261:619-29.

4. Fretland ÅA, Dagenborg VJ, Bjørnelv GMW, et al. Laparoscopic versus open resection for colorectal liver metastases: The OSLO-COMET randomized controlled trial. Ann Surg 2018;267:199-207.

5. Robles-Campos R, Lopez-Lopez V, Brusadin R, et al. Open versus minimally invasive liver surgery for colorectal liver metastases (LapOpHuva): a prospective randomized controlled trial. Surg Endosc 2019;33:3926-36.

6. Fretland ÅA, Dagenborg VJ, Waaler Bjørnelv GM, et al. Quality of life from a randomized trial of laparoscopic or open liver resection for colorectal liver metastases. Br J Surg 2019;106:1372-80.

7. Beppu T, Wakabayashi G, Hasegawa K, et al. Longterm and perioperative outcomes of laparoscopic versus open liver resection for colorectal liver metastases with propensity score matching: a multi-institutional Japanese study. J Hepatobiliary Pancreat Sci 2015;22:711-20.

8. Allard MA, Cunha AS, Gayet B, et al. Colorectal Liver Metastases-French Study Group. Early and Long-term Oncological Outcomes After Laparoscopic Resection for Colorectal Liver Metastases: A Propensity Score-based Analysis. Ann Surg 2015;262:794-802.

9. Zhang XL, Liu RF, Zhang D, et al. Laparoscopic versus open liver resection for colorectal liver metastases: A systematic review and meta-analysis of studies with propensity score-based analysis. Int J Surg 2017;44:191-203. 
10. Syn NL, Kabir T, Koh YX, et al. Survival Advantage of Laparoscopic Versus Open Resection For Colorectal Liver Metastases: A Meta-analysis of Individual Patient Data From Randomized Trials and Propensity-score Matched Studies. Ann Surg 2020;272:253-65.

11. Lonjon G, Boutron I, Trinquart L, et al. Comparison of treatment effect estimates from prospective nonrandomized studies with propensity score analysis and randomized controlled trials of surgical procedures. Ann Surg 2014;259:18-25.

12. Fretland AA, Sokolov A, Postriganova N, et al. Inflammatory Response After Laparoscopic Versus Open Resection of Colorectal Liver Metastases: Data From the Oslo-CoMet Trial. Medicine (Baltimore) 2015;94:e1786

13. Lyu X, Qiao W, Li D, et al. Impact of perioperative blood transfusion on clinical outcomes in patients with colorectal liver metastasis after hepatectomy: a meta-analysis. Oncotarget 2017;8:41740-8.

14. Montalti R, Berardi G, Laurent S, et al. Laparoscopic liver resection compared to open approach in patients with colorectal liver metastases improves further resectability: oncological outcomes of a case-control matched-pairs analysis. Eur J Surg Oncol 2014;40:536-44.

15. van der Poel MJ, Barkhatov L, Fuks D, et al. Multicentre propensity score-matched study of laparoscopic versus open repeat liver resection for colorectal liver metastases. Br J Surg 2019;106:783-9.

16. Kobayashi S, Beppu T, Honda G, et al. Survival benefit and indications for adjuvant chemotherapy for resected colorectal liver metastases-a Japanese nationwide survey. J Gastrointest Surg 2020;24:1244-60.

17. Kawai T, Goumard C, Jeune F, et al. Laparoscopic liver resection for colorectal liver metastasis patients allows patients to start adjuvant chemotherapy without delay: a propensity score analysis. Surg Endosc 2018;32:3273-81.

18. Cheng Y, Zhang L, Li H, Wang L, et al. Laparoscopic versus open liver resection for colorectal liver metastases: a systematic review. J Surg Res 2017;220:234-46.

19. Halls MC, Cipriani F, Berardi G, et al. Conversion for Unfavorable Intraoperative Events Results in Significantly Worse Outcomes During Laparoscopic Liver Resection: Lessons Learned From a Multicenter Review of 2861 Cases. Ann Surg 2018;268:1051-7.

20. Yamashita S, Venkatesan AM, Mizuno T, et al. Remnant Liver Ischemia as a Prognostic Factor for Cancer-Specific Survival After Resection of Colorectal Liver Metastases. JAMA Surg 2017;152:e172986.
Cite this article as: Akahoshi S, Beppu T, Mori Y, Kinoshita K, Sato N, Kikuchi K. Does laparoscopic liver resection for colorectal liver metastases provide a true survival benefit compared with open liver resection? HepatoBiliary Surg Nutr 2020;9(5):657-660. doi: 10.21037/hbsn.2020.01.01 